\title{
Self-esteem as a Moderator of the Effects of Happiness, Depression, and Hostility on Suicidality Among Early Adolescents in Korea
}

\author{
Yeun-Soon Choi', Hee Kyoung Shin², Dae-Yong Hong ${ }^{1}$, Jang-Rak Kim', Yune-Sik Kang', Baekgeun Jeong ${ }^{1}$, Ki Soo Park', \\ Key Hyo Lee ${ }^{3}$ \\ 'Department of Preventive Medicine, and Institute of Health Science, Gyeongsang National University College of Medicine, Jinju, Korea; \\ ${ }^{2}$ Department of Nursing, Inje University, Busan, Korea; ${ }^{3}$ School of Public Health, Inje University, Busan, Korea
}

Objectives: The purpose of this study was to investigate the role of self-esteem as a moderator of the factors influencing suicidality among middle-schoolers.

Methods: Moderated multiple regression analysis was applied to assess the influence of happiness, depression, and hostility on suicidality and to determine the degree to which self-esteem served as a moderator of those relationships. Data were collected from 268 students at a middle school in Busan, Korea, using a self-administered structured questionnaire.

Results: Happiness, depression, and hostility had significant direct effects on suicidality. Self-esteem showed no direct effect, but had a significant moderating effect on the relationship between hostility and suicidal behavior.

Conclusions: These results suggest that various interventions, such as counseling programs, should be designed to alleviate hostility and depression and to enhance happiness and self-esteem among early adolescents.

Key words: Suicidality, Hostility, Self-esteem, Early adolescents

\section{INTRODUCTION}

Youth suicide is an important public health problem that is urgent to solve in our community. According to World Health Organization estimates, suicide is among the top 3 leading causes of death among youth worldwide [1]. In the Republic of Korea (hereafter Korea), the leading cause of death among

Received: April 30, 2018 Accepted: January 2, 2019

Corresponding author: Key Hyo Lee, PhD

School of Public Health, Inje University, 75 Bokji-ro, Busanjin-gu,

Busan 47392, Korea

E-mail: keyinje@gmail.com

This is an Open Access article distributed under the terms of the Creative Commons Attribution Non-Commercial License (http://creativecommons.org/licenses/by$\mathrm{nc} / 4.0 /$ ) which permits unrestricted non-commercial use, distribution, and reproduction in any medium, provided the original work is properly cited. youth has been suicide since 2007 [2]. According to the 2018 cause-of-death data from Statistics Korea (http://kostat.go.kr/ portal/korea/index.action), the number of suicides per 100000 population for youth between 10 and 19 declined from 5.5 in 2011 to 4.2 in 2015, 4.9 in 2016, and 4.7 in 2017 [3]. Despite a decrease in the overall suicide rate from 2011 to 2017, suicide rates in teenagers and twenties is still a serious social problem due to the fact that the decline trend is not clear.

Youth suicide needs to be addressed with especially careful consideration, since adolescence is when the foundations for lifelong mental health are laid, and appropriate interventions should be implemented by applying preventive measures based on systematic educational programs in schools. Several domestic studies have been conducted on youth suicide.

However, most domestic studies on youth suicide have focused on identifying and evaluating risk factors for suicidal 
behaviors. Prior research has shown that several factors are intertwined in the phenomenon of youth suicide, including genetic characteristics, personality and ways of responding, depression or anxiety, and forms of psychopathology such as psychological anxiety, accumulated stress, delinquency, school performance, family conflicts, peer conflicts, relationship difficulties, and physical disorders [4-7].

These various and complicated risk factors for suicide are very challenging to understand on an individual level. Youth suicide is especially challenging to comprehend since youth tend not to disclose personal information to authority figures or adults. Therefore, identifying factors that significantly influence the likelihood of suicide is important in order to easily recognize suicidal symptoms in youth. For example, if easilyobservable risk factors are established, it would be possible to provide interventions for youth at high risk for suicide. This study focused on happiness, depression, and hostility as factors influencing youth suicide. These variables are representative aspects of the psychological status of youth that parents, teachers, and friends can easily observe.

For happiness, subjective judgments, which refer to how a person feels about his or her life, are more important than objective standards [8]. According to the 2016 Korean Children and Adolescents' Happiness Index, in a comparison with 22 Organization for Economic Cooperation and Development nations conducted by the Korean Sofa Organization in 2016, the subjective happiness of Korean adolescents was ranked at the bottom; furthermore, this has been the case since 2009 [9]. A 2006 study of the raw data of an online survey on adolescent health behavior suggested that happiness influenced the likelihood of youth suicide [10]. Furthermore, an analysis of the factors influencing suicidal ideation among youth in metropolitan areas that focused on youth mental health facilities showed that youth with suicidal ideation reported lower degrees of subjective happiness than those without suicidal ideation [11]. These findings collectively imply that happiness should be considered as a positive predictor of youth suicide.

Several studies have shown depression to be the risk variable with the highest mean effect size on youth suicide $[6,7,10-12]$. However, another report stated that there was no correlation between depression and suicidality in youth, in contrast to adults [13].

Furthermore, there is a close correlation between youth suicide and hostility [4]. Hostility has a significant influence on suicidal ideation in youth [12]. There is also a research report- ing that hostility, which refers to the presence of defiant, negative, and aggressive behavior, is more related to youth suicide than depression [13].

In addition, possible moderating variables should be considered with respect to the aforementioned influencing factors. Under similar situations or conditions, some youth overcome their hardships without despair or frustration, whereas others choose to commit suicide. Moderating variables could help explain these discrepancies in outcomes.

Therefore, this study focused on self-esteem as a moderating variable of the effects of happiness, depression, and hostility on youth suicide. Previous studies have shown that self-esteem is a significant predictor of suicidality $[6,14]$, and it also has been studied as a moderating variable of the relationships of stress, depression, and abuse experiences with suicidal ideation $[6,15,16]$. However, no study has simultaneously analyzed happiness, depression, and hostility as influencing factors and examined the moderating effect of self-esteem on those relationships. For this reason, the present study significantly broadens the research agenda on the role and function of self-esteem in youth suicide.

More specifically, this study empirically examined the effects of happiness, depression, and hostility as influencing factors on suicidal ideation, planning, and attempts among early adolescents, and explored self-esteem as a moderator of those relationships. Through these findings, this study will contribute to the development and implementation of suicide prevention programs.

This study builds upon previous studies in two aspects. First, it narrowed the scope of the research by choosing the significant influencing factors of happiness, depression, and hostility as independent variables, since these are relatively straightforwardly observable symptoms, in contrast to the excessively broad range of influencing factors enumerated in previous research. Second, it examined the mediating effect of self-esteem, which plays a significant role in explaining why some youths make different choices under highly similar circumstances, but has not been sufficiently dealt with as a moderating variable in previous studies on youth suicide.

\section{METHODS}

\section{Study Participants}

Data were collected from middle school students in grades 7 through 9 at a middle school in Busan Metropolitan City 
through a self-administered, structured questionnaire survey. The survey was conducted as follows. A letter explaining the content and purpose of questionnaire was discussed with the teacher in charge. This letter was sent to each family a few days before the survey was administered, and after receiving consent forms from the parents, the survey was conducted. The questionnaire survey was conducted among a total of 276 students, with the exception of those who did not want to participate. In the final data analysis, 268 surveys were used, excluding those with either no answers or inappropriate answers. The study received Institutional Review Board (IRB) approval from the Ethics Committee of Inje University Hospital (IRB No. 2-1041024-AB-N-01-20150123-HR-145).

\section{Operational Definitions and Measures Socio-demographic characteristics}

Socio-demographic characteristics included sex, grade, siblings, school records, subjective economic level, and religious activities. School performance and subjective economic level were measured by using a 5-point Likert scale.

\section{Influencing factors}

Happiness is defined by subjective judgements of how a person feels about his or her life, rather than objective standards [8]; hence, it is a concept that can be measured by subjectively evaluating the degree to which an individual's life is in accordance with his or her internal criteria. Happiness is predicted to lower the likelihood of suicidal ideation, planning, and attempts. The study used a modified version of the instrument developed by Han [14], which originally contained 45 items, and was adjusted to include items suitable for youth. The survey consisted of 9 items, including 'My family is happy, 'I have a good relationship and I am popular,' I I am at ease and I enjoy my leisure time,' I am physically healthy and I am satisfied with my appearances,' 'I am satisfied with living with my family,' 'There are many things to be grateful for in my life,' 'I am satisfied with my overall school life including my school environment and atmosphere, 'I plan my life goals by myself,' and 'I always keep in mind the most important thing at the present moment.' These items were measured using a 5-point Likert scale. The total score of happiness ranged from 9 (lowest) to 45 (highest), with higher scores indicating a greater degree of happiness. The Cronbach alpha (0.902) was high.

Depression is defined as an emotional state involving worry, gloom, a sense of failure, loss, lack of energy, and insignifi- cance [17]. It is predicted to increase the likelihood of suicidal ideation, planning, and attempts. For this study, the Center for Epidemiologic Studies Depression Scale (CES-D) developed by Radloff [18] was modified by the author of the present study to be more suitable for the study population, middle school students, within the scope of the meaning of CES-D. The survey consisted of 7 items, including 'Recently, I have been frequently annoyed,' 'I feel gloomy even though my family or friends help me,' I am afraid of thinking about the future,' Recently, I have had difficulty sleeping,' 'There are times when I feel unhappy,'I tend not to enjoy my life,' and 'There are times I feel lonely. These items were measured using a 5-point Likert scale. The total score of depression ranged from 7 (lowest) to 35 (highest), with higher scores indicating a greater degree of depression. The Cronbach alpha (0.831) was high.

Hostility is defined as an emotional state involving hatred, cynicism, and aggression due to negative social relationships with others [19]. It is predicted to increase the likelihood of suicidal ideation, planning, and attempts. To measure hostility, this study used a modified version of the survey used in a study of Korean youth health by the National Youth Policy Institute of Korea [20]. The survey consisted of 4 items including 'I sometimes feel an overwhelming sense of anger,' I feel urges to assault or harm someone,' I feel urges to smash things,' and 'I sometimes yell or throw things.' These items were measured using a 5-point Likert scale. The total score of hostility ranged from 4 (lowest) to 20 (highest), with higher scores indicating a greater degree of hostility. The Cronbach alpha (0.893) was high.

\section{Moderator: self-esteem}

Self-esteem is a factor that influences physical, emotional, and behavioral problems of youth, and is defined as a positive self-assessment on ordinary days [21]. Therefore, it is predicted to moderate the relationships of other influencing variables on suicidal ideation, planning, and attempts. To measure self-esteem, this study used a modified version of Korean translation version of Rosenberg's self-esteem scale [22] and by the National Youth Policy Institute of Korea [20]. The survey consisted of 6 items, including 'I think I have a good nature,'I am capable of working well with the majority of people,' 'I don't have anything to be proud of,' I am satisfied with myself in general,' I sometimes feel like I am useless,' and 'I sometimes think that I am not a good person.' These items were measured using a 5-point Likert scale. Reverse scoring was 
used for the negative items. The total self-esteem score ranged from 6 (lowest) to 30 (highest), with higher scores indicating a higher degree of self-esteem. The Cronbach alpha (0.830) was high.

\section{Dependent variables}

Suicide has been conceptualized in numerous different ways by many scholars; of particular note, however, O'Connell et al. [19] suggested that suicide involves a series of process including suicidal ideation, suicidal plans, suicide attempts, and completed suicide as part of the model of suicidality. From suicidal ideation to completed suicide, predictors can vary by path; hence, this study divided suicide into 3 processes for analysis. Completed suicide was not analyzed in this study.

Many domestic studies have used the translated version of the Suicidal Ideation Questionnaire (SIQ) developed by Reynold [23] and the Scale for Suicidal Ideation (SSI) developed by Beck et al. [17]. This study used those 2 survey instruments for reference. However, to minimize the number of items regarding suicide, in order to make the survey as suitable as possible for youth, this concept was measured using a single item for each of the 3 processes involved in suicide. This choice was made to minimize the likelihood of confusing responses from the study population, since the SIQ and SSI repeatedly ask items with similar content and consist of various types of items. In the mental health section of the Korea National Health and Nutrition Examination Survey, suicidal ideation is measured with the single item of 'Over the past year, have you ever thought about dying?' for the same reason.

The study used a 5-point Likert scale for this measure, in accordance with the use of a 5-point scale in the SIQ and a 3-point scale in the SSI. In contrast, the corresponding item in the Korea National Health and Nutrition Examination Survey uses a categorical response format, with an answer of either yes or no. However, a 5-point scale was chosen in this study instead of a binary scale since it minimizes data loss.

\section{Suicidal ideation}

Suicidal ideation refers to thoughts about engaging in suicidal behavior, including temporary thoughts such as 'There is no point in living' or 'I want to die.' The study used a single item ('Sometimes I want to die for no reason.') that was scored on a 5-point Likert scale.

\section{Suicidal plans}

Suicidal plans refers to a behavioral level beyond suicidal ideation, which refers to simply wanting to die, but does not directly involve self-injurious behavior. The concept of suicidal plans includes making a will, organizing one's personal belongings, and gathering information about suicide. To measure suicidal plans, a single item ('I have composed a suicide note.') was used, with a 5-point Likert scale.

\section{Suicide attempts}

Suicide attempts involve translating suicidal ideation and planning into a specific action. Theses can include acts performed to draw attention or threaten someone, without a real intention of dying, as well as failed attempts to achieve the goal of suicide. To measure suicide attempts, we used a single item ('I have attempted suicide in the past.') that was scored on a 5-point Likert scale.

\section{Data Analysis}

The collected data were analyzed using SPSS version 21 (IBM Corp., Armonk, NY, USA). The socio-demographic characteristics of the study population were examined using descriptive statistics and a reliability analysis was conducted on the measured variables with multiple items. Pearson correlation analysis was used to examine the correlations among study variables. Lastly, hierarchical moderated regression analysis was used to assess the direct effects of the 3 predictors (happiness, depression, and hostility) on the dependent variables and the effects of self-esteem as a moderating variable.

In the moderated regression analysis, mean centering of the values of the variables was applied to diminish multicollinearity in relation to hypothesis testing for significance among the predictors. Mean centering means not including zero as a measurement value of the predictors; hence, it is a helpful way to compensate for a lack of significance in data interpretation [24].

Tests for multicollinearity in the moderated regression analysis showed that the tolerance between the independent variable and moderator variable was over 0.5 . The variance inflation factor (VIF) was less than 2, and the maximum value of the condition index was 24.644 , which satisfied the general standard that a tolerance under 0.1 , a VIF over 10 , and a condition index over 30 suggest the problem of multicollinearity [25]. 


\section{RESULTS}

\section{Socio-demographic Characteristics of the Study} Population and Descriptive Analysis of the Variables

The socio-demographic characteristics of the study population are shown in Table 1. The most common responses were as follows: females (52.6\%); ninth grader (44.0\%); high grades in school (26.1\%); an average economic level (60.1\%); and no religious activity (79.5\%).

In the descriptive statistics of the study variables, which all had values between 1 to 5 , the mean values and standard deviations were as follows: happiness ( $3.85 \pm 0.74)$, depression $(2.63 \pm 0.94)$, hostility $(2.09 \pm 1.02)$, self-esteem $(3.54 \pm 0.68)$,

Table 1. Socio-demographic characteristics of the study population

\begin{tabular}{llc}
\hline Characteristics & Categories & $\mathbf{n}(\%)$ \\
\hline Sex & Male & $127(47.4)$ \\
Grade & Female & $141(52.6)$ \\
& First & $92(34.3)$ \\
& Second & $58(21.6)$ \\
Siblings & Third & $118(44.0)$ \\
& No & $39(14.5)$ \\
School performance & Yes & $229(85.4)$ \\
& Very low & $12(4.5)$ \\
& Low & $49(18.3)$ \\
& Middle & $122(45.5)$ \\
& High & $70(26.1)$ \\
Economic level & Very high & $15(5.6)$ \\
& Very low & $0(0.0)$ \\
& Low & $14(5.2)$ \\
& Middle & $161(60.1)$ \\
Total & High & $83(31.0)$ \\
& Very high & $10(3.7)$ \\
Religious activities & No & $213(79.5)$ \\
& Yes & $55(20.5)$ \\
& & $268(100)$ \\
& &
\end{tabular}

suicidal ideation (2.26 \pm 1.21$)$, suicidal plans $(1.37 \pm 0.80)$, and suicide attempts (1.44 \pm 0.92$)$.

Among the dependent variables, suicidal ideation had the highest mean score (2.26). Happiness showed the highest mean score (3.85) among the independent variables. Self-esteem, a moderating variable, showed a relatively high mean (3.54) (Table S1).

\section{Correlations Between the Study Variables}

Simple correlations between the study variables are shown in Table 2. Among the predictors, hostility (with a positive relationship) showed the most significant correlations with suicidal ideation and suicidal plans. Depression (with a positive relationship) was a less significant variable, and happiness (with a negative relationship) was the least significant. Furthermore, suicide attempts, rather than hostility, had the most significant correlation with suicidal plans.

\section{Empirical Model Testing}

\section{Factors influencing suicidal ideation and the moderating effect of self-esteem}

Table 3 demonstrates the results of the hierarchical moderated regression analysis of the 3 factors that influenced suicidal ideation and the moderating effect of self-esteem. Model 1 , in the first stage of the moderated regression analysis, is a regression model that only included the independent variables, and it showed significant effects of depression $(B=0.11, p<$ $0.05)$ and hostility $(B=0.96, p<0.01)$ on suicidal ideation. Model 2 , in the second stage, is a regression model that added a moderating variable (self-esteem) to model 1 , and self-esteem did not show a significant effect on suicidal ideation. Model 3 , in the third stage, is a regression model that added interactions between the independent variables and the moderating variable, and it showed no significant effect of self-esteem, the moderating variable, on the relationships of the 3 predictors with the dependent variables.

Table 2. Correlations between study variables

\begin{tabular}{|c|c|c|c|c|c|c|}
\hline & Happiness & Depression & Hostility & Self-esteem & Suicidal ideation & Suicidal plans \\
\hline Depression & $-0.49 * * *$ & & & & & \\
\hline Hostility & $-0.38^{* * *}$ & $0.55^{* * *}$ & & & & \\
\hline Self-esteem & $0.61^{* * *}$ & $-0.60 * * *$ & $-0.36^{* * *}$ & & & \\
\hline Suicidal ideation & $-0.31^{* * *}$ & $0.51^{* * *}$ & $0.84^{* * *}$ & $-0.34^{* * *}$ & & \\
\hline Suicidal plans & $-0.28^{* * *}$ & $0.28^{* * *}$ & $0.36^{* * *}$ & $-0.18^{* * *}$ & $0.24^{* * *}$ & \\
\hline Suicide attempts & $-0.37^{* * *}$ & $0.41^{* * *}$ & $0.44^{* * *}$ & $-0.29 * * *$ & $0.32^{* * *}$ & $0.68^{* * *}$ \\
\hline
\end{tabular}

${ }^{* * *} p<0.001$. 


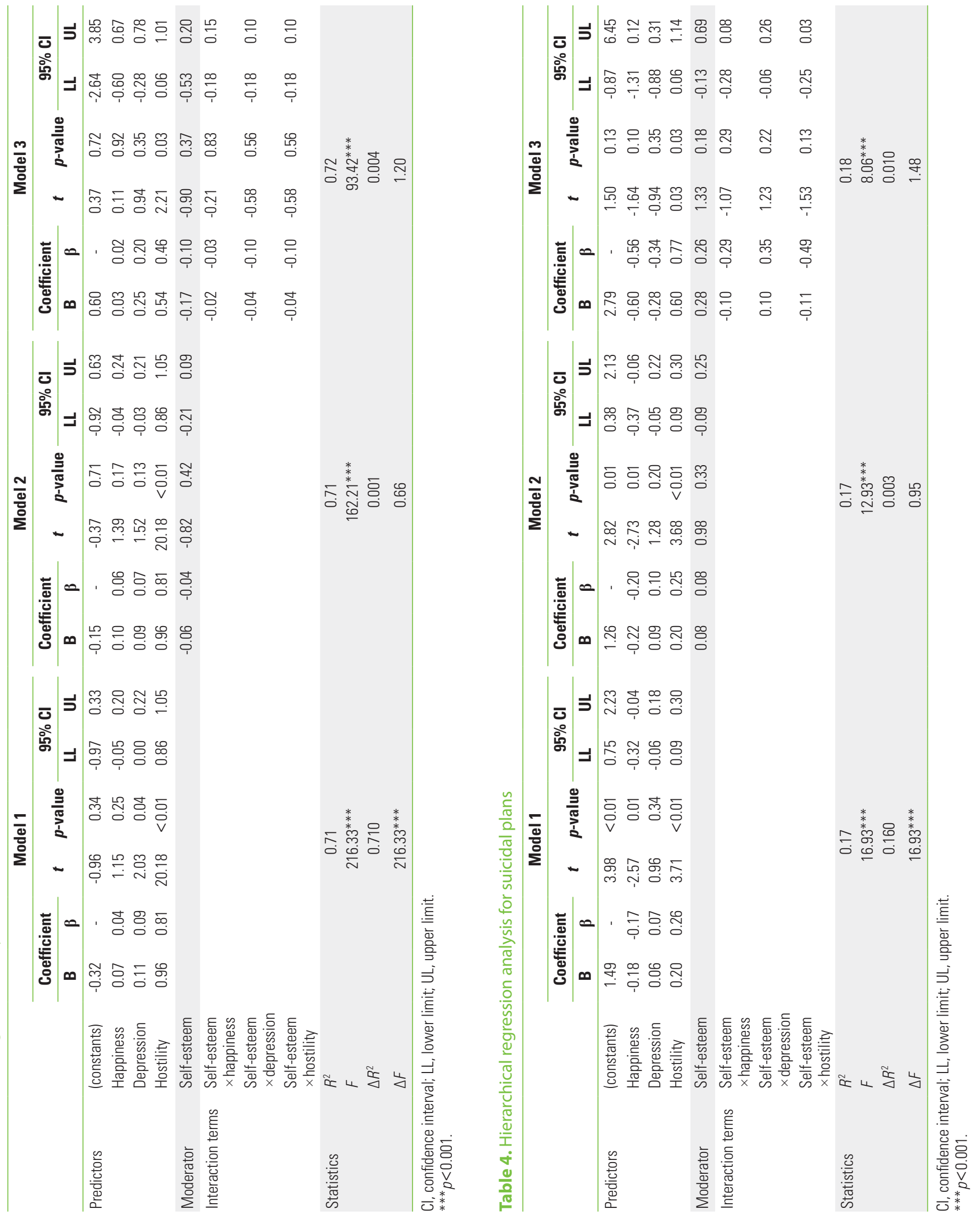




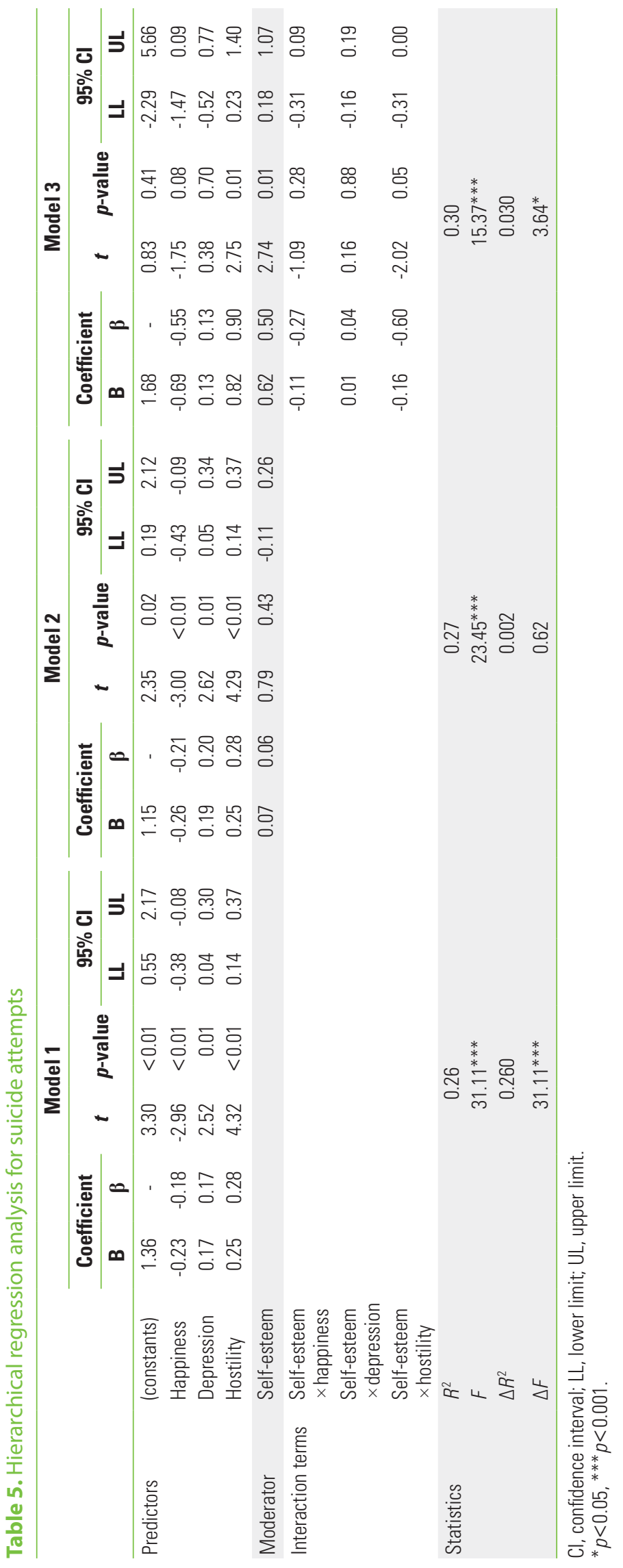

\section{Factors influencing suicidal plans and the moderating effect of self-esteem}

The results of the hierarchical moderated regression analysis of the effects of the 3 influencing factors on suicidal plans and the moderating effect of self-esteem are shown in Table 4. Model 1 showed significant effects of happiness $(B=-0.18, p<$ $0.05)$ and hostility $(B=0.20, p<0.01)$ on suicidal plans. Model 2 showed no significant effect of self-esteem as a moderating variable for suicidal plans. Model 3 showed no significant effect of self-esteem, the moderating variable, on the relationships between the 3 predictors and the dependent variables.

\section{Factors influencing suicide attempts and the moderating effect of self-esteem}

The results of the hierarchical moderated regression analysis of the effects of the 3 influencing factors on suicide attempts and the moderating effect of self-esteem are shown in Table 5. Model 1 showed significant direct effects of the 3 influencing factors. Hostility $(\mathrm{B}=0.25, p<0.01)$ had the most significant influence on suicide attempts. Happiness $(B=-0.23, p<0.01)$ had less significance than hostility, and depression $(B=0.17$, $p<0.05$ ) had the least significance. Model 2 showed no significance of self-esteem as a moderating variable of these relationships. In model 3 , the interaction between self-esteem and hostility $(B=-0.16, p<0.05)$ was significant, confirming the moderating effect of self-esteem.

The moderated regression equation used to create a graph to visualize the moderating effect of self-esteem on the relationship between hostility and suicide attempts is shown below:

$$
\begin{aligned}
\mathrm{Y}(\text { suicide attempt })= & 1.36+0.25 \mathrm{X} \text { (hostility) }+ \\
& 0.05 \mathrm{M} \text { (self-esteem) }-0.15 \mathrm{XM}
\end{aligned}
$$

A graph of the moderating effect made using the above moderated regression equation is shown in Figure S1. If the value of the moderating variable is low, the slope is near 0 . However, as the value of the moderating variable increases, the slope becomes steeper. This shows the diminishing moderating effect of hostility on suicide attempts.

\section{DISCUSSION}

The purpose of this study was to provide basic data to support preventive measures against youth suicide by assessing 
the direct effects of happiness, depression, and hostility on suicidality and the moderating effect of self-esteem on those relationships among middle-school students. The main findings are discussed below.

First, the results of the univariate analysis of the correlations between study variables showed a significant relationship between the influencing factors and the dependent variables. Namely, suicidal ideation, suicidal plans, and suicide attempts became more common as happiness decreased, whereas the opposite relationship was found for depression and hostility.

In the results of the univariate correlation analysis, it is noteworthy that suicide attempts - the nearest proxy variable for completed suicide-had a significant correlation with suicidal ideation and suicidal plans. Of particular note, suicidal plans showed the closest correlation with suicide attempts. Youth with experiences of suicidal ideation and suicidal plans had a higher likelihood of attempting suicide. These findings can be understood in a similar context to that of previous research results $[26,27]$ that showed more suicidal behaviors among youth who had previously attempted suicide and that more suicide attempts occurred among youth with extensive suicidal ideation. The findings of this study were also similar to those of a previous study that found that participants with experiences of suicidal ideation had a $>30$ times greater risk of attempting suicide [10].

Second, among the 3 influencing factors, depression and hostility showed significant direct effects on suicidal ideation; happiness and hostility showed significant direct effects on suicidal plans; and happiness, depression, and hostility showed significant direct effects on suicide attempts. It is noteworthy that hostility showed the strongest positive direct effect on the 3 dependent variables of suicidal ideation, suicidal plans, and suicide attempts.

Youth sometimes experience difficulties controlling their anger in conflicts or stressful situations and may show defiant or negative attitudes, since their cognitive and emotional development is not fully complete. Such hostility generally includes aggression. Titelman et al. [28] asserted that suicide is a completion of an attack towards oneself by projecting aggressive transference.

Such hostility is a more serious risk factor for youth than for adults, and Gispert et al. [13] asserted that anger and hostility are risk factors that are more closely related with youth suicide than depression. Domestic studies of youth have shown that factors related to hostility, including impulsivity, predicted the risk of suicide better than depression and despair [29]. Impulsive and aggressive tendencies or behaviors, which are derived from hostility, are important predictive factors of suicidal behaviors among youth [12].

The result that happiness had a significant negative influence on suicidal ideation is in accordance with previous findings [11]. This result suggests that it is important to find and reduce triggering factors of suicidality, but also that making efforts to strengthen happiness would be another good way to diminish suicidality from the perspective of positive psychology.

Furthermore, depression had a significant influence on suicidal ideation and suicide attempts. However, the results of this study are distinct from those of previous research, in that the magnitude of the influence of depression was smaller than that of hostility and happiness, and depression showed no significant effects on suicidal plans. In previous studies, depression was found to be a frequent psychological problem among youth, and it is considered to be a risk factor that has a powerful influence on suicidal ideation among youth $[8,9,11$, 23]. Many studies have investigated the relationship between depression and suicidal ideation, but relatively few have explored the relationship of depression with suicidal plans or suicide attempts. Therefore, the relationship of depression with suicidal plans and suicide attempts should be thoroughly studied in the future.

Third, self-esteem showed a significant negative correlation with the dependent variables, including suicidal ideation, suicidal plans, and suicide attempts, in the univariate correlation analysis, but it showed no significant direct effect in the multiple regression analysis. However, self-esteem functioned as a moderator of the relationship between hostility, a strong influencing factor, and suicide attempts. The finding of our study that self-esteem had no direct effect on suicidal ideation, suicidal plans, or suicide attempts, but had a significant moderating effect on suicide attempts through its interaction with hostility, suggests that it is more valid to study self-esteem as a moderating variable than as an independent variable.

It should be taken into consideration that, as shown in Tables 3-5, the regression analysis of model 2 showed no significance influence for self-esteem; however, model 3 in Table 5 showed that self-esteem had a significant positive influence, unlike what was theoretically predicted. This result shows that the added interactions in model 3 functioned as suppressor variables, increasing the regression coefficient value of the 
self-esteem variable, thereby increasing its statistical significance and changing the directionality of the relationship. The existence of suppression indicates that the simple effect of self-esteem was not significant [30].

The result that self-esteem had no significant direct effect on suicidal ideation, suicidal plans, and suicide attempts is supported by some results from previous studies [16,31]. However, other studies have shown that self-esteem is a direct risk factor, since lower self-esteem increased the possibility of suicidal ideation $[5,15,32]$. These results regarding the direct effect of self-esteem on suicidal ideation are uncertain, and should further investigated in the future.

The finding of this study that self-esteem functioned as a moderator, reducing the influence of hostility on suicide attempts, is significant. Many studies have investigated the influence of hostility on youth suicide $[14,29,33]$, but none have simultaneously explored hostility and self-esteem. Previous research into the moderating or mediating effects of self-esteem has included studies of its interaction with stress [15,32], a study on the influence of depression as a mediator on suicidal ideation [31], and a study on self-esteem as a moderator of suicidal ideation among youth with experiences of abuse [16].

The findings of the present study regarding the moderating effect of the interaction between self-esteem and hostility correspond with previous findings, including a study of low self-esteem as a contributor to crime and violence and as a cause of hostility and aggression targeting middle school students [34]. Furthermore, an approximately 50\% decrease in unruly behavior and verbal or physical aggression was found after implementing a self-esteem program in a school [35]. Low self-esteem led to expressions of negative attitudes and aggression among youth, since they handled conflict situations inappropriately [33]. In contrast, youth with high self-esteem thought of themselves as worthy people, hence reducing hostility; thus, self-esteem can function as a mechanism to reduce the risk of extreme behaviors such as suicide attempts. Furthermore, self-esteem is related to happiness in general. Therefore, it is expected to have some effect on suicide prevention. In consideration of the effects of self-esteem, multilateral intervention measures should be prepared to improve the self-esteem of youth as part of efforts to weaken the link between hostility and suicide attempts.

In summary, the results of this study suggest that active intervention measures, such as counseling, should be developed and used to reduce hostility among youth, considering the powerful influence of hostility on youth suicide. Furthermore, mental health programs should be implemented to increase happiness by targeting youth. For happiness, subjective judgements of how a person feels about his or her life are more important than objective standards, so interventions to improve mental health should be considered [10]. Additionally, program development and implementation could be important intervention measures to improve self-esteem among youth.

For the interpretation and generalization of the results of this study, several limitations need to be considered. First, since the study targeted ordinary middle-school students, not a high-risk group, problems such as suicidal ideation, suicidal plans, and suicide attempts were not very prevalent. Second, this study was based on the analysis of data from a sample of middle-school students in Busan Metropolitan City, meaning that there is a limitation in the generalizability of the study results due to the risk of selection bias. Third, the suicide-related items in the questionnaire were designed to be simple, considering the characteristics of the study population of relatively young middle-school students. However, the measurement scale could be limited since the items for other variables were adopted from general-use measurement instruments. Fourth, this was a cross-sectional study, making it difficult to clearly validate the temporal relationship between the dependent variables and the influencing factors or moderating variables. Fifth, although the simple effect of self-esteem was not significant in this study, considering the suppression effect, the follow-up studies to verify the simple and moderating effect of self-esteem should be conducted for a clearer conclusion.

\section{SUPPLEMENTARY MATERIALS}

Supplementary materials are available at https://www.jpmph. org/.

\section{CONFLICT OF INTEREST}

The authors have no conflicts of interest associated with the material presented in this paper.

\section{ORCID}

Yeun-Soon Choi https://orcid.org/0000-0002-6200-2875

Hee Kyoung Shin https://orcid.org/0000-0002-9377-507X

Dae-Yong Hong https://orcid.org/0000-0002-0735-5571 
Jang-Rak Kim https://orcid.org/0000-0002-8191-5071

Yune-Sik Kang https://orcid.org/0000-0002-3404-279X

Baekgeun Jeong https://orcid.org/0000-0002-1655-5436

Ki Soo Park https://orcid.org/0000-0001-5571-3639

Key Hyo Lee https://orcid.org/0000-0001-7939-8776

\section{REFERENCES}

1. World Health Organization. Mental health: suicide data [cited 2017 Sep 11]. Available from :http://www.who.int/mental_ health/prevention/suicide/suicideprevent/en/.

2. Korean Statistical Informations Service. Juvenile statistics; 2017 [cited 2017 Sep 11]. Available from: http://kosis.kr/upsHtml/ online/downSrvcFile.do?PUBCODE=YG\&FILE_NAME=/YG/13. zip\&SEQ $=423$ (Korean).

3. Korea National Statistical Office. Annual report on the cause of death statistics 2018 [cited 2018 Feb 14]. Available from: http://kostat.go.kr/portal/korea/kor_nw/2/6/2/index.board?b mode $=$ read\&bSeq $=218 \& a S e q=363268 \&$ pageNo $=1 \&$ row um $=10 \&$ navCount $=10 \&$ currPg $=\&$ sTarget $=$ title\&sTxt (Korean).

4. Bridge JA, Goldstein TR, Brent DA. Adolescent suicide and suicidal behavior. J Child Psychol Psychiatry 2006;47(3-4):372-394.

5. Park JY. The effects of risk and protective factors on adolecent suicide. Korea J Youth Stud 2009;16(10):249-272 (Korean).

6. Kim BY, Lee CS. A meta-analysis of variables related to suicidal ideation in adolescents. J Korean Acad Nurs 2009;39(5):651661 (Korean).

7. Moon DK, Kim YH. A meta-regression analysis on related triggering variables of adolescents' suicidal ideation. Korean J Couns 2011;12(3):945-964 (Korean).

8. Diener E. Subjective well-being. Psychol Bull 1984;95(3):542575.

9. Korean Sofa Organization. Korean children juvenile happiness index; 2016 [cited 2017 Sep 11]. Avalilable from: https://www. korsofa.org:44315/bbs/board.php?bo_table=data\&wr_id = 74 (Korean).

10. Park E. The influencing factors on suicide attempt among adolescents in South Korea. J Korean Acad Nurs 2008;38(3):465473 (Korean).

11. Kim DA. Meta-analysis on variables related to suicide attempt among Korean adolescents [dissertation]. Seoul: Ewha Womans University; 2017 (Korean).

12. Kim M, Kim J. A study on the influencing mental health factors to suicidal ideation in adolescents. Korean J Youth Stud 2013; 20(12):267-293 (Korean).
13. Gispert M, Davis MS, Marsh L, Wheeler K. Predictive factors in repeated suicide attempts by adolescents. Psychiatr Serv 1997; 38(4):390-393.

14. Han SM. The effects of degree of home-environmental wellbeing on adolescent's emotional intelligence, social intelligence and happiness [dissertation]. Incheon: Inha University; 2011 (Korean).

15. Sin YH, Park SY. Effects of adolescents' interpersonal relationship stress on suicidal ideation: examination of the moderating role of self-esteem. J Youth Welf 2016;18(1);235-259 (Korean).

16. Kim JY, Lee K. Effect of adolescents' abuse experience on suicidal ideation: focused on moderated mediation effect of selfesteem on depression and anxiety. J Korean Acad Nurs 2015; 45(5):752-760 (Korean).

17. Beck AT, Kovacs M, Weissman A. Assessment of suicidal intention: the Scale for Suicide Ideation. J Consult Clin Psychol 1979; 47(2):343-352.

18. Radloff LS. The CES-D scale: a self-report depression scale for research in the general population. Appl Psychol Meas 1997; 1(3):385-401.

19. O'Connell H, Chin AV, Cunningham C, Lawlor BA. Recent developments: suicide in older people. BMJ 2004;329(7471): 895-899.

20. Choi IJ. 2010 Korean Youth Health Survey; 2011 [cited 2018 Feb 14]. Available from: http://lib.nypi.re.kr/pdfs/2010/45.pdf (Korean).

21. Coopersmith S. Antecedents of self-esteem. San Francisco: Freeman; 1967, p. 3-9.

22. Jun BJ. Self-esteem: a test of its measurability. Yonsei Nonchong 1974;11(1):107-130 (Korean).

23. Reynolds WM. SIQ, Suicidal Ideation Questionnaire: professional manual. Odessa: Psychological Assessment Resources; 1988, p. 10-11.

24. lacobucci D, Schneider MJ, Popovich DL, Bakamitsos GA. Mean centering helps alleviate "micro" but not "macro" multicollinearity. Behav Res Methods 2016;48(4):1308-1317.

25. Myers RH. Classical and modern regression with applications. 2nd ed. Belmont: Duxbury Press; 1990, p. 384-385.

26. Bonner RL, Rich AR. Toward a predictive model of suicidal ideation and behavior: some preliminary data in college students. Suicide Life Threat Behav 1987;17(1):50-63.

27. Kim HS, Kim BS. Verification of the structural relationship model of suicidal ideation to its related variables. Korean $J$ Couns Psychol 2008;20(2):201-209 (Korean). 
28. Titelman D, Nilsson A, Svensson B, Karlsson H, Bruchfeld S. Suicide-nearness assessed with PORT, the Percept-genetic Object-Relation Test: a replication and a reliability study. Bull Menninger Clin 2011;75(4):295-314.

29. Go HJ, Kim DJ, Lee HP. A validation study of the suicide probability scale for adolescents (SPS-A). J Korean Neuropsychiatr Assoc 2000;39(4):680-690 (Korean).

30. Thompson FT, Levine DU. Examples of easily explainable suppressor variables in multiple regression research. Mult Linear Regression Viewp 1997;24(1):11-13.

31. Kim IG, Jo NJ. The validation of structural models on adolescents' suicidal ideation. Korean J Couns 2006;7(4):1189-1202
(Korean).

32. Lee SH, Chun JS. The influence of stress on suicidal ideation among male and female adolescents: focusing on the moderating effects of self-esteem. Ewha J Soc Sci 2012;28:173-202 (Korean).

33. Pyon BK, Kang SK. A study of adolescents' self-esteem and hostility. Korean J Youth Stud 2002;9(3):269-291 (Korean).

34. Kaplan HB. Self-attitudes and deviant behavior. Soc Forces 1976;54(4):788-801.

35. Kernis MH. Toward a conceptualization of optimal self-esteem. Psychol Inq 2003;14(1):1-26. 